\title{
A Preliminary Investigation of Longitudinal Changes in Speech Production over 18 Months in Young Children with Cerebral Palsy
}

\author{
Jimin Lee Katherine C. Hustad \\ Department of Communicative Disorders and Waisman Center, University of Wisconsin-Madison, \\ Madison, Wisc., USA
}

\section{Key Words}

Acoustic analysis · Developmental disorders • Dysarthria • Speech intelligibility

\begin{abstract}
Objective: This study examined longitudinal change in speech intelligibility, vowel space, and word duration over 18 months among children with cerebral palsy (CP) who varied in the severity of their speech motor involvement. The study also examined relationships among variables at each time point. Method: Twenty-two children with CP participated in the study (mean age $=50$ months at the first time point). Speech samples were collected at four time points that were 6 months apart. Children were separated into four severity groups based on intelligibility scores. Results: Change over time varied by severity. Children with CP who did not have speech motor involvement and children in the mild group showed gains in intelligibility, but no changes in vowel space area or word duration. Children in the moderate group showed no significant change, and children in the severe group showed increased vowel space and decreased word durations. Significant positive correlations between intelligibility and vowel space were noted at each time point for data pooled across all children. Conclusion: Children showed dif-
\end{abstract}

ferent patterns of change over time in intelligibility, vowel space, and word duration based on their speech motor abilities. The relationship between intelligibility and vowel space across severity groups was constant, suggesting a robust relationship between these variables.

Copyright $\odot 2013$ S. Karger AG, Basel

It is well established that children with cerebral palsy (CP) often have communication problems [1]. Although data are somewhat limited, studies suggest that dysarthria may be the most common communication problem in those with CP $[2,3]$. Dysarthria almost always has a negative impact on speech intelligibility, which in turn affects functional communication, social participation, and quality of life. However, little is known about speech characteristics of children with CP, and even less is known about the longitudinal course of speech development in these children. Thus, the prognosis for speech development in children with CP is unclear at the outset.

An understanding of the nature, rate, and potential limits of advancement in speech development in children with CP has important consequences for intervention decisions, and thus is the focus of this research. Considerable efforts have sought to characterize the speech

\section{KARGER}

E-Mail karger@karger.com www.karger.com/fpl
(C) 2013 S. Karger AG, Basel

1021-7762/13/0651-0032\$38.00/0 
of adults with dysarthria secondary to various etiologies. Studies have examined perceptual as well as acoustic features of speech, and methodologies for characterizing temporal and spectral acoustic parameters have been well established for the study of adult dysarthria [4]. In adults with $\mathrm{CP}$, studies indicate that speech deficits seem to involve all speech subsystems (respiration, phonation, velopharyngeal function, and articulation) to some extent. However, of all speech subsystems, articulation appears to have received the most attention for speakers with CP. Research has demonstrated that vowel space tends to be reduced [5], vowel productions are often inaccurate $[6,7]$, and abnormalities in the timing and range of tongue movements tend to occur [8]. In children with dysarthria secondary to CP, far less is known. Studies have examined primarily older children, and research suggests that there may be a strong relationship between vowel space and intelligibility [9]. However, it is unknown whether this relationship extends to younger children with CP. Such a relationship, if present, may suggest a relatively easy-to-obtain but important quantitative indicator of speech motor control ability in young children with CP.

Although we have a general sense of the nature of speech production problems associated with $\mathrm{CP}$, we do not know how speech changes with growth and development. Expectations for speech development can be inferred from the literature on studies of speech production in young typically developing children. Studies examining spectral characteristics of vowels in typically developing children suggest that first and second formant frequency (F1 and F2, respectively) values and segment durations gradually decrease until about 18 years of age and that variability also decreases with age $[10,11]$. It is possible that abnormality in vowel development could be a good index of overall speech motor control problems because vowels are mastered very early in normal development.

Considerable efforts to understand developmental changes in gross and fine motor performance in children with CP have been underway for some time. Results of such studies suggest that the limit of development is negatively correlated with severity such that children with more severe gross motor deficits reach maximum skill levels at earlier ages than children with less severe deficits [12]. Although the progression of speech motor development may be different from the progression of gross motor development, we were interested in examining whether there might be differential patterns of change in the progression of speech development based on the nature of the child's speech-motor disorder. For the reasons reviewed above, measures of the acoustic vowel space and word duration were deemed good measurement candidates to reveal changes in speech production ability.

The goal of the present study was to examine changes over time in speech intelligibility, vowel space, and word duration in children with CP. The following research questions were addressed: (1) How do vowel space, word duration, and intelligibility change in children with $\mathrm{CP}$ within operationally defined profile groups across an 18-month longitudinal period? (2) Are there significant relationships between vowel space area and speech intelligibility within any of the four time points, when data are pooled across all children?

\section{Methods}

\section{Participants}

Children with CP

A total of 22 children (11 male; 11 female) with CP participated in this study. All children were part of a larger longitudinal project on communication development in children with CP [3]. For the present study, inclusion criteria required that children: (1) have a medical diagnosis of CP; (2) have hearing abilities within normal limits as determined by hearing screening or formal audiological evaluation; (3) have completed four data collection sessions as part of the larger project, and (4) be able to produce singleword utterances in imitation. All children from the larger study who met these criteria at the time of data analysis were included. The average chronological age of children was: 50 months (SD = 9.8) at the first time point, 55 months $(\mathrm{SD}=9.9)$ at the second time point, 61 months $(S D=9.9)$ at the third time point, and 67 months $(\mathrm{SD}=9.9)$ at the fourth time point.

Because children were extremely heterogeneous, they were separated into profile groups following the classification model described by Hustad et al. [3]. This model comprises eight different groups which differ primarily based on the presence or absence of speech motor involvement (SMI) and secondarily based on language and cognitive involvement. Preliminary validity for this model has been established for clinical assignment into profile groups which were statistically differentiable based on quantitative analyses of speech and language measures. Importantly, the variables that best discriminated among groups were speech rate and vowel space. In the present study, we were interested in examining change over time in speech variables only. Therefore, we separated children into profile groups as described by Hustad et al. [3] based only on speech production data, and children who were unable to speak were excluded from this study.

A certified speech-language pathologist separated children into two groups: those with SMI, and those who had no clinical evidence of speech motor involvement (NSMI). SMI was defined as clinical evidence of motor impairment in any one or more of the speech subsystems (articulation, phonation, resonation, respiration) that could be observed perceptually via visual and/or auditory modalities during speech or at rest. NSMI was defined by the absence of such evidence in any of the speech subsystems. 
Table 1. Demographics of children with CP by profile group

\begin{tabular}{|c|c|c|c|c|}
\hline & \multicolumn{4}{|l|}{ Groups } \\
\hline & SMI-severe $(\mathrm{n}=4)$ & SMI-moderate $(\mathrm{n}=4)$ & SMI-mild $(\mathrm{n}=5)$ & NSMI $(n=9)$ \\
\hline \multicolumn{5}{|l|}{ Mean chronological } \\
\hline age at time 1 (range) & 53.2 months (43-65) & 45.0 months (34-59) & 50.5 months $(40-60)$ & 49.5 months $(29-60)$ \\
\hline Male/female ratio & $2 / 2$ & $2 / 2$ & $1 / 4$ & $3 / 6$ \\
\hline \multicolumn{5}{|l|}{ Type of CP } \\
\hline Spastic & 2 & 3 & 3 & 8 \\
\hline Dyskinetic & 1 & 0 & 1 & 0 \\
\hline Ataxic & 0 & 1 & 0 & 0 \\
\hline Mixed & 1 & 0 & 0 & 0 \\
\hline Unknown & 0 & 0 & 1 & 1 \\
\hline \multicolumn{5}{|l|}{ Mean \% intelligibility } \\
\hline across time points (SD) & $7.9(5.4)$ & $31.6(15.2)$ & $61.5(14.0)$ & $73.0(14.0)$ \\
\hline
\end{tabular}

A second certified speech-language pathologist (the second author), who was blinded to the classifications made by the first speech-language pathologist, also classified the children based on videotaped observations of spontaneous speech. Agreement between the two sets of classifications was $95 \%$ ( 21 of 22 children).

Among the 22 children with CP, 13 children had clinical evidence of SMI. They were further subdivided into severity groups based on average intelligibility scores across the four time points. Four children were assigned to the SMI-severe group (word intelligibility <20\%), 4 to the SMI-moderate group (word intelligibility between 21 and 50\%), and 5 to the SMI-mild group (word intelligibility between 51 and 100\%). The remaining 9 children had $\mathrm{CP}$, but no clinical evidence of SMI; intelligibility scores were not considered for classification purposes in the NSMI group. Mean demographic characteristics of children by profile group, including age, sex, type of $\mathrm{CP}$, and intelligibility score are presented in table 1 .

\section{Listeners}

Two listeners were randomly assigned from a consecutive series of volunteers to hear each child at each time point (4 time points $\times 22$ children $\times 2$ listeners $=176$ listeners $)$. Each listener heard only 1 child at one time point to prevent a learning effect. Inclusion criteria required that each listener: (1) pass a pure-tone hearing screening at $25 \mathrm{~dB} H \mathrm{HL}$ for $250 \mathrm{~Hz}, 500 \mathrm{~Hz}, 1 \mathrm{kHz}, 4 \mathrm{kHz}$, and $6 \mathrm{kHz}$ bilaterally; (2) be between 18 and 45 years of age; (3) have no more than incidental experience listening to or communicating with persons having communication disorders; (4) be a native speaker of American English, and (5) have no identified language, learning, or cognitive disabilities per self-report. There was no criterion for sex of listeners.

To control for potential variability among the small number of listeners for each child, we examined the mean intelligibility difference between the 2 listeners who heard each child. If the average difference in word intelligibility scores was more than $10 \%$, data were obtained from a third listener and the two intelligibility data points that differed by $10 \%$ or less were used. This occurred in 5 instances and in each instance data from a third listener were within $10 \%$ of one of the other 2 listeners.

\section{Materials and Procedures}

Speech samples collected for this study were used to obtain both acoustic and intelligibility data. Single-word stimuli from the Test of Children's Speech (TOCS) [15] were produced by each child using delayed imitation. Samples were recorded using digital equipment in a sound-attenuating suite. From the corpus of 38 different TOCS words produced by each child, eight words were selected based on their phonetic characteristics (to permit reliable measurement of the acoustic variables of interest). Words selected for acoustic analysis were: sheet, seat; hoot, boot; top, hot; bad, hat. Four repetitions of each target word were attempted with each child. However, at the first and second time points, on average, three analyzable productions of each target word were obtained. Of these, the production that was judged to be the best was used for intelligibility analyses, while all repetitions were used for acoustic analyses.

Speech samples were played for listeners in a sound-attenuating suite via an in-house computer program. The peak audio output level was calibrated to approximately $75 \mathrm{~dB}$ SPL at the listeners' location. Listeners heard each word one time and were instructed to type an orthographic transcription of each word. To prevent an order effect the sequence of presentation of stimulus words was randomized; thus, no 2 listeners heard the stimulus words in the same order.

\section{Analysis of Data: Speech Acoustics and Intelligibility}

Speech Acoustics

Temporal and vowel spectral measures were obtained from digital speech samples. A wideband spectrographic display in TF32 [16] and established measurement criteria [13] were used to obtain the measures that follow. (1) Duration of each of the eight target words was obtained by measuring the time between the onset of audible or visible acoustic energy associated with production of the first phoneme of the stimulus word and the offset of acoustic energy associated with production of the last phoneme of the stimulus word. (2) First and second formant frequencies (F1 and F2, respectively) for each of the eight target words containing corner vowels were obtained using a 30 -ms window at the temporal midpoint of each of the four corner vowels. Spectrogram analysis bandwidth was adjusted for each child based on his/her F0. 
Linear predictive coding was used to generate formant tracks which were hand-corrected based on visual inspection, as necessary. Formant values were used to calculate vowel space following Johnson et al. [17].

Interjudge reliability was obtained by having a second judge make all acoustic measures on $10 \%$ of the data. To calculate interjudge reliability, Pearson product-moment correlation coefficients and absolute differences were examined for the first and second set of measures. Correlations ranged from 0.94 to 0.99 . The mean absolute difference for the two sets of measures for each variable was as follows: word duration $=28 \mathrm{~ms}$; vowel duration $=$ $9 \mathrm{~ms} ; \mathrm{F} 1=27 \mathrm{~Hz} ; \mathrm{F} 2=32 \mathrm{~Hz}$. Values were within an acceptable range following Kent et al. [13].

Intelligibility

Orthographic transcriptions were scored by counting the number of transcribed words that matched the target words phonemically. Misspellings and homonyms were accepted as correct, as long as all phonemes in the spoken version of the transcribed words matched the target words. Mean intelligibility scores for each child were obtained by averaging intelligibility data from each of the 2 listeners per child. Data are reported as the percentage of words identified correctly.

\section{Experimental Design and Analysis}

Due to the small number of participants in each severity group, nonparametric statistical tests were utilized. Analyses addressing longitudinal questions employed the Friedman test to examine overall differences across time points for each of three dependent variables (vowel space, word duration, intelligibility) within each severity group. Differences between time points within each group were examined descriptively because of the small number of children within each group. Correlations among dependent variables across all children were calculated for each time point and change over time in the relationships among variables was examined descriptively. Due to the exploratory nature of this study, tests with $\mathrm{p}=0.05$ were considered significant.

\section{Results}

Descriptive data for children in the four severity groups for each time point are shown in table 2. Figure 1 shows vowel quadrilateral plots for each time point and profile group. Descriptive results suggest that patterns of change over time differed by variable and by severity group. For children in the SMI-severe group, descriptive data suggest that vowel space decreased over time, while word duration and intelligibility increased over time. Inferential statistics revealed that the patterns of decreasing vowel space (TS $=8.10 ; \mathrm{p}=0.044)$ and increasing word duration (TS $=9.30 ; p=0.026)$ were significant for children in the SMI-severe group.

For children in the SMI-moderate group, descriptive data suggest that vowel space, word duration, and intelligibility increased over time. Inferential statistics re-
Table 2. Mean data for each dependent variable by severity group and time point

\begin{tabular}{llll}
\hline & $\begin{array}{l}\text { Vowel space } \\
\mathrm{Hz}^{2}\end{array}$ & Word duration & $\begin{array}{l}\text { Word intelli- } \\
\text { ms } \\
\text { gibility (all } \\
\text { TOCS words) }\end{array}$ \\
& & & \\
\hline SMI-severe group & & \\
Time 1 & $380,032(103,382)$ & $669.60(300.37)$ & $4.30(2.65)$ \\
Time 2 & $353,730(185,807)$ & $830.05(393.92)$ & $7.85(6.35)$ \\
Time 3 & $116,380(134,083)$ & $893.35(481.65)$ & $7.83(4.96)$ \\
Time 4 & $182,148(122,481)$ & $1,139.08(194.10)$ & $11.53(6.36)$ \\
\hline SMI-moderate group & & \\
Time 1 & $353,557(225,574)$ & $401.78(85.90)$ & $22.68(17.61)$ \\
Time 2 & $563,703(216,289)$ & $571.83(113.21)$ & $34.18(20.95)$ \\
Time 3 & $443,989(155,555)$ & $615.93(47.37)$ & $27.18(7.62)$ \\
Time 4 & $636,799(267,656)$ & $569.98(68.61)$ & $42.30(7.04)$ \\
\hline SMI-mild group & & \\
Time 1 & $812,518(95,461)$ & $691.24(83.11)$ & $50.48(3.86)$ \\
Time 2 & $831,959(112,446)$ & $664.72(48.86)$ & $60.96(4.95)$ \\
Time 3 & $832,433(156,022)$ & $707.38(201.95)$ & $61.42(9.18)$ \\
Time 4 & $756,418(150,260)$ & $618.76(78.51)$ & $73.06(7.72)$ \\
\hline NSMI group & & & \\
Time 1 & $908,685(275,460)$ & $567.84(65.61)$ & $63.71(18.03)$ \\
Time 2 & $933,887(257,258)$ & $596.18(74.37)$ & $71.46(13.96)$ \\
Time 3 & $932,380(311,569)$ & $586.26(59.10)$ & $77.21(9.76)$ \\
Time 4 & $907,432(193,302)$ & $598.16(81.72)$ & $79.69(8.58)$ \\
\hline
\end{tabular}

Standard deviations are given in parentheses.

vealed that there were no significant changes in any of the variables over time.

For children in the SMI-mild group, descriptive data suggest that intelligibility increased over time and that change in the other variables was highly variable over time. Inferential statistics indicated that the pattern of increasing intelligibility was the only significant finding for children in the mild group ( $\mathrm{TS}=13.56 ; \mathrm{p}=0.004$ ).

For children in the NSMI group, descriptive data suggest that vowel space and intelligibility increased over time; however, there was considerable variability in word duration over time. Inferential statistics indicated that the pattern of increasing intelligibility was the only significant finding for children in the NSMI group (TS = 13.79; $\mathrm{p}=0.003$ ).

Correlations among pairs of the dependent variables with children pooled across severity groups for each time point revealed moderate-strong correlations at each of the four time points for intelligibility and vowel space $(\mathrm{r}=0.721$, time $1 ; 0.659$, time $2 ; 0.734$, time $3 ; 0.757$, time 4 ; 


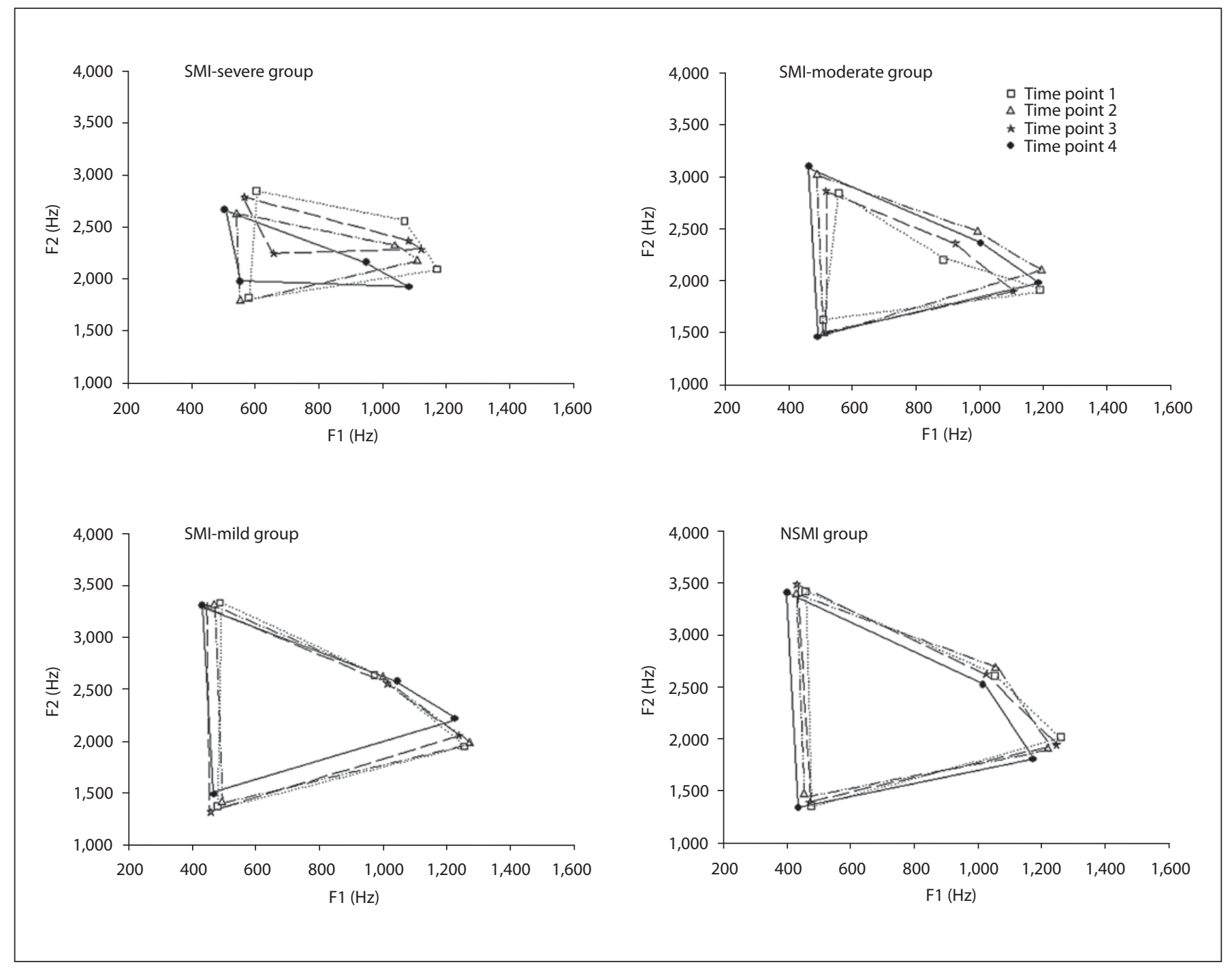

Fig. 1. Vowel quadrilaterals at each time point by severity group.

$p<0.001$, respectively). A scatterplot showing the relationship between vowel space and intelligibility at the first time point, which is also illustrative of data for the other time points, is shown in figure 2.

\section{Discussion}

Findings from this study suggest that there was considerable variability among the profile groups with regard to performance on the intelligibility, vowel space, and word duration variables, and there was surprisingly little systematic change over the 18-month time frame of this study. It was notable, however, that there was a strong and consistent relationship between intelligibility and vowel space for each of the time points across all children. Findings, their relation to extant literature on speech development and clinical implications are discussed below.

\section{Longitudinal Change within Profile Groups}

Research on intelligibility and acoustics of children's speech is somewhat limited and has primarily involved cross-sectional studies that have varied in the subtleties of methodology. However, intelligibility is expected to increase as children develop. Generally, studies suggest that 


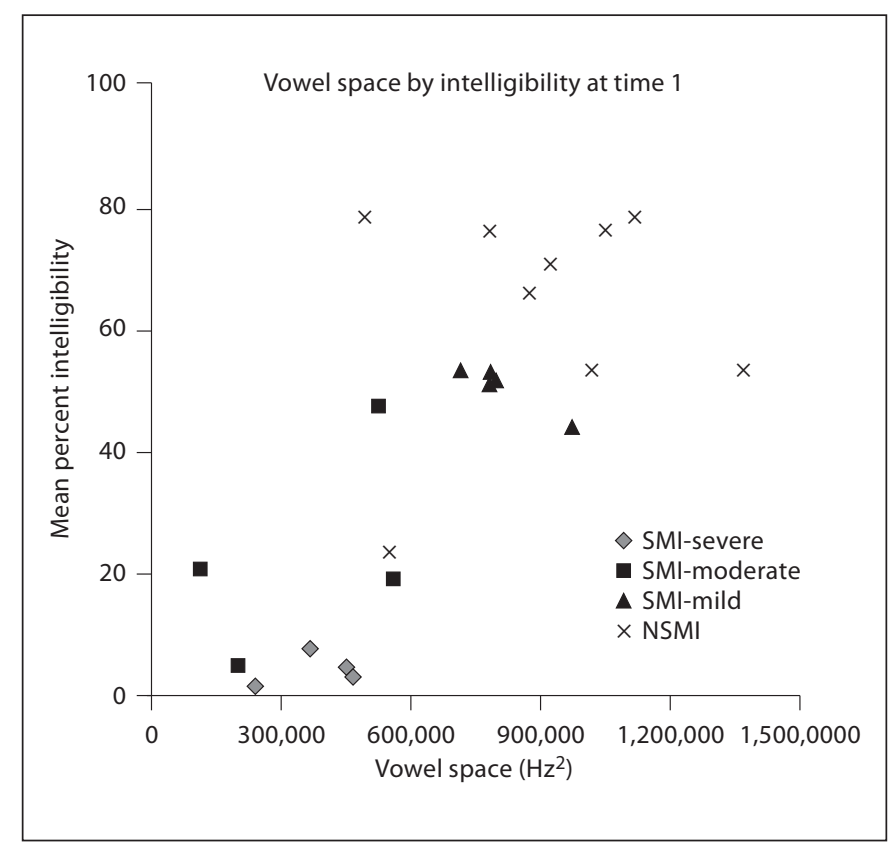

Fig. 2. Correlation between intelligibility and vowel space for the first time point across all children.

vowel space area should decrease, with formants shifting to lower frequency ranges between the ages of 3 and 4 years up through adolescence and adulthood $[10,11,18]$. However, findings from one study [18] suggest that for children between the ages of 3 and 7 years, vowel space may fluctuate because of complex interactions between developmental improvements in speech motor control combined with anatomical growth during this critical speech development period. Studies examining changes in duration measures suggest that generally duration should decrease between the ages of 5 years and adulthood [10].

Children in the NSMI group had a diagnosis of CP, but did not have clinically observable signs of SMI. Therefore, it is reasonable to expect that children in the NSMI group should have performance that is comparable to that of typically developing children on the same measures. However, this was the case only for intelligibility, which increased approximately $15 \%$ over the 18 -month time period. The magnitude of this increase was grossly consistent with age expectations [19, 20]. However, it is difficult to explain why children in the NSMI group did not show the expected changes on the acoustic variables. One explanation may be methodological differences across studies, and the lack of normative longitudinal data in the literature. Also, in the present study, children in the NSMI group spanned an age range of 29 months at each of the longitudinal time points (youngest child 31 months; oldest child 60 months at the first time point). It is possible that the heterogeneity in chronological age among children in this group obfuscated the ability to observe change, particularly if change was tied to narrow age bands. Alternatively, the 18-month longitudinal window in the present study may not have been long enough to observe changes in acoustic measures, regardless of age. Finally, children in the NSMI group may have had subtle subclinical speech production differences that made them different from typically developing children without CP. A control group of typically developing children, age- and sex-matched with children in the NSMI group, is necessary to further explore this possibility.

Children in the SMI-mild group showed a pattern of change that was very similar to children in the NSMI group, with significant improvements of approximately $20 \%$ in intelligibility, and no changes in the other speech variables. However, it should be noted that mean intelligibility scores were consistently lower on average than for children in the NSMI group. Children in the SMI-mild group might benefit from speech intervention that aims to accelerate gains in developmental speech production skills or supplemental augmentative and alternative communication strategies to enhance intelligibility and to advance independent communication and social participation.

Children in the SMI-moderate group showed considerable variability both among individual children and among time points. Children in this group appeared to be somewhat static with regard to their speech development, showing no real change over time. A longer time window and/or a larger number of children may reveal different findings. Nonetheless, results suggest that any progress made by children with moderate dysarthria secondary to CP may be very gradual.

Children in the SMI-severe group showed patterns of change that were different from children in the other groups and from typically developing expectations. Specifically, these children showed significantly increased word durations and decreased vowel space with time. The pattern of decreasing vowel space with time is generally consistent with age expectations, however, the small size of the vowel space, the associated distorted shape of vowel quadrilaterals (fig. 1), and the very low intelligibility scores clearly suggest that these children differ from their peers in other groups in both quantitative and qual- 
itative ways. The increases in word duration over time observed for children in this group reflect a trend in the opposite direction of developmental expectations. One possible explanation may be that developmental phonetic advancement results in increased duration for children with severe SMI. Phonetic features of speech were not evaluated in this study, but perceptual phonetic analyses may be an interesting avenue for further investigation. Another potential explanation for the increases in word duration over time may relate to changes in muscle tone of the articulators. One study examining the gastrocnemius-soleus muscle (located in the lower leg) in children with $\mathrm{CP}$ reported increased muscle tone up to age 4 and then decreased muscle tone up to age 12 [21]. In the gross motor literature, the longitudinal course of movement function seems to be more favorable for children with less severe motor impairments [12]. Therefore, the pattern of increased word duration may be more apparent for children with severe dysarthria. However, these are speculations based on previous gross motor literature in children with CP. Gross motor and speech motor control are different [22]. Hence, the developmental course of articulator muscle tone in children with $\mathrm{CP}$ and its relation to acoustic characteristics of speech needs to be investigated.

\section{Relationship between Vowel Space and Intelligibility}

Of particular interest was the strong and consistent relationship between vowel space and speech intelligibility at each of the time points (fig. 2). This finding was consistent with observations from adults with dysarthria, and findings of the present study tended to show stronger correlations between vowel space and intelligibility than pre- vious studies have demonstrated in adults with dysarthria [23]. Additional research is necessary to examine a wider array of acoustic variables such as F2 trajectory and velopharyngeal variables to further understand contributors to intelligibility that may be potential intervention targets.

\section{Limitations and Future Directions}

The results of the present study should be interpreted with caution. Findings are based on a relatively small number of participants who varied in age. Data reflect a limited number of speech tokens taken from single-word productions from each child. Finally, the longitudinal window of this study was short relative to the prolonged period in which speech development occurs in children. These limitations point to future research directions that employ a larger sample, increased homogeneity with regard to the age of children examined, connected speech samples, and a longer time span to characterize longitudinal speech development in children with CP.

\section{Acknowledgments}

The authors thank the children with CP and their families who participated in this research. We also thank Andrea Nett, who completed the reliability analyses on acoustic data as well as the graduate and undergraduate students at the University of Wisconsin-Madison who assisted with data collection and data reduction. This study was funded by grants K23DC007114 and R01DC009411 from the National Institute on Deafness and Other Communication Disorders, National Institutes of Health (United States Department of Health and Human Services). Support was also provided by the Waisman Center Core Grant P30HD0335 from the National Institute of Child Health and Human Development, National Institutes of Health.

\section{References}

$\checkmark 1$ Bax M, Tydeman C, Flodmark O: Clinical and MRI correlates of cerebral palsy: the European Cerebral Palsy Study. JAMA 2006; 296:1602-1608

2 Sigurdardottir S, Vik T: Speech, expressive language, and verbal cognition of preschool children with cerebral palsy in Iceland. Dev Med Child Neurol 2010;53:74-80.

- 3 Hustad KC, Gorton K, Lee J: Classification of speech and language profiles in 4-year-old children with cerebral palsy: a prospective preliminary study. J Speech Lang Hear Res 2010;53:1496-1513.

4 Kent R, Weismer G, Kent J, Rosenbek J: Toward phonetic intelligibility testing in dysarthria. J Speech Hear Disord 1989;54:482499.
5 Liu H-M, Tsao F-M, Kuhl PK: The effect of reduced vowel working space on speech intelligibility in Mandarin-speaking young adults with cerebral palsy. J Acoust Soc Am 2005;117:3879-3889.

-6 Platt LJ, Andrews G, Howie P: Dysarthria of adult cerebral palsy. II. Phonemic analysis of articulation errors. J Speech Hear Res 1980; 23:41-55.

7 Platt LJ, Andrews G, Young M, Quinn PT: Dysarthria of adult cerebral palsy. I. Intelligibility and articulatory impairment. J Speech Hear Res 1980;22:28-40.

8 Kent R, Netsell R: Articulatory abnormalities in athetoid cerebral palsy. J Speech Hear Disord 1978;43:353-373.
-9 Higgins CM, Hodge MM: Vowel area and intelligibility in children with and without dysarthria. J Med Speech Lang Pathol 2002; 10:271-277.

10 Lee S, Potamianos A, Narayanan S: Acoustics of children's speech: developmental changes of temporal and spectral parameters. J Acoust Soc Am 1999;105:1455-1468.

- 11 Vorperian HK, Kent RD: Vowel acoustic space development in children: a synthesis of acoustic and anatomical data. J Speech Lang Hear Res 2007;50:1510-1545.

12 Rosenbaum PL, Walter SD, Hanna SE, Palisano RJ, Russell DJ, Raina P, et al: Prognosis for gross motor function in cerebral palsy: creation of motor development curves. JAMA 2002;288:1357-1363. 
13 Kent RD, Weismer G, Kent JF, Vorperian HK, Duffy JR: Acoustic studies of dysarthric speech: methods, progress, and potential. J Commun Disord 1999;32:141-186.

-14 Selby JC, Robb MP, Gilbert HR: Normal vowel articulations between 15 and 36 months of age. Clin Ling Phonet 2000;14: 255-265.

15 Hodge M, Daniels J: TOCS+ Intelligibility Measures. Edmonton, University of Alberta, 2007.

16 Milenkovic P: TF32. Madison, University of Wisconsin-Madison, 2002.
Johnson K, Flemming E, Wright R: Response to Whalen et al. Language 2004;80:646-648.

18 Eguchi S, Hirsh IJ: Development of speech sounds in children. Acta Otolaryngol 1969; 257:1-51.

19 Morris SR, Wilcox KA, Schooling TL: The preschool speech intelligibility measure. Am J Speech Lang Pathol 1995;4:22-28.

20 Gordon-Brannan M, Hodson BW: Intelligibility/severity measurements of prekindergarten children's speech. Am J Speech Lang Pathol 2000;9:141-150.

21 Hagglund G, Wagner P: Development of spasticity with age in a total population of children with cerebral palsy. BMC Musculoskelet Disord 2008;9:150.
22 Kent RD: Models of speech motor control: implications from recent developments in neurophysiological and neurobehavioral science; in Maassen B, Kent RD, Peters HFM, van Lieshout PHHM, et al (eds): Speech Motor Control in Normal and Disordered Speech. Oxford, Oxford University Press, 2004, pp 3-28.

23 Turner G, Tjaden K, Weismer G: The influence of speaking rate on vowel space and speech intelligibility for individuals with amyotrophic lateral sclerosis. J Speech Hear Res 1995;38:1001-1013. 